\title{
TRAYECTORIAS, CONEXIONES Y CONVERGENCIAS TEÓRICAS EN LA ARQUEOLOGÍA
}

Dante Angelo ${ }^{\text {a }}$

\section{Oteando el horizonte...}

La arqueología, comúnmente, es definida como la ciencia que estudia el pasado a través de sus restos materiales. Entre los desafíos de la arqueología, el proveer una narrativa coherente y completa de la historia de la humanidad, es quizás el mayor. En su libro Historia del Pensamiento Arqueológico, uno de los textos más relevantes y aún vigentes en la historia de la disciplina, Bruce Trigger (1989) provee algunas de las claves más importantes para entender el proceso de "cientifización» de la disciplina. La trayectoria de la disciplina, nos dice Trigger, atraviesa etapas de formación en la que los primeros aficionados intentaron otorgar a la arqueología su carácter y que luego se embarcarían en un esfuerzo de consolidación científica de una práctica disciplinar supeditada a la historia. Los debates que seguirían a la formación del pensamiento arqueológico han sido sumarizados con detalle en distintos volúmenes y han sido comúnmente presentados a modo de batallas (por ejemplo: la nueva arqueología vs. la historia cultural; la arqueología postprocessual $v s$. la arqueología procesual/nueva arqueología; objetividad $v$ s. relativismo, ciencia $v s$. humanismo) ( $c f .:$ Binford 1983, 1987; Hodder (ed.) 1982; Kohl 1993; Shanks y Tilley 1987; Yoffee y Sherratt 1993).

Recientemente, el arqueólogo escandinavo Kristian Kristiansen, emulando el esquema Kunhniano de los paradigmas de la ciencia, propuso entender esta trayectoria en términos de revoluciones científicas; según Kristiansen (2014), luego de un desgaste producido por debates que caracteriza como quasi estériles, estaríamos asistiendo — vía recientes avances tecnológicos: Big Data, arqueometría, genética y otros-, a una tercera revolución científica en arqueología. Casi paralelamente, un connotado grupo de colegas estadounidenses (y un británico) escribían en PNAS, el portal oficial de la Academia Nacional de Ciencias de su país, buscando definir cuales eran los desafíos científicos más importantes para la arqueología. Este distinguido ensamble de colegas buscaba responder a la consulta de la NSF (siglas en inglés de la Fundación Nacional de Ciencias), quizás la institución más importante en ciencia e investigación en Estados Unidos, sobre la forma de cómo priorizar sus inversiones en términos de infraestructura tecnológica que pueda favorecer

\footnotetext{
a Universidad de Tarapacá

Correo electrónico: dangeloz@gmail.com
} 
el avance de la investigación científica de la arqueología. Los resultados del análisis logrado por dicho grupo señalaban, entre otras cosas: otorgar prioridad a las preguntas generalizadoras (o big questions), enfatizando temas de causalidad y la producción de modelos predictivos (Kintigh et al. 2014).

Haciendo un paréntesis, me gustaría introducir algunos elementos que hasta aquí he mantenido fuera de la discusión, lo cual, a su vez, me permitirá introducir el tema central que me interesa desarrollar en este texto: las contribuciones teóricas a la arqueología desde ámbitos latinoamericanos. Reflexionando sobre el panorama teórico en Latino América, hace ya casi dos décadas, Gustavo Politis (2003) decía que nuestra arqueología se caracterizaba ampliamente por una mirada muy tradicional del pasado que, a pesar de cierta sofisticación metodológica, la adopción de modelos ecológico-ambientales, y un énfasis analítico y comparativo, todavía se ocupaba más en producir datos que en formular propuestas teóricas. Antes, como observa Politis (2003; Politis y Pérez Gollán 2004), la celebrada Arqueología Social Latinoamericana había buscado plantear una lectura del pasado que, inspirada en el marxismo y el materialismo histórico, reflejara las contradicciones de las sociedades americanas en el pasado.

Esperando clarificar mi argumento, aunque consciente de lo que significa enunciar una crítica a la notable contribución de colegas como Luis G. Lumbreras en una revista como el Boletín de Arqueología PUCP (la casa de la arqueología peruana), voy a arriesgarme a decir algo más respecto de este programa. En términos teóricos, a mi parecer, a pesar de cierta crítica epistemológica, la Arqueología Social Latinoamericana no se distanció significativamente de propuestas funcionalistas y sistémicas en los que el modelo hipotético deductivo y los esquemas evolucionistas (tribu, cacicazgo, estado) campeaban para explicar las sociedades del pasado (ver por ejemplo: Gándara 1987, 1992; Lumbreras 1989, 2007; Sanoja 1984; Bate 2002). Y si bien, «criticaban la subordinación de la arqueología en el continente, cuestionaban la epistemología histórica-cultural y reivindicaban una postura política radical en relación con los problemas sociales» (Politis y Pérez-Gollán 2004: 354), escasamente se pronunciaban contra la epistemología colonial y cientificista de la arqueología (cf. Pagán-Jimenez 2004). Contemplada comúnmente con profunda nostalgia, en su momento, este programa pretendía ofrecer un pensamiento más propio, «más latinoamericano», en contraposición a corrientes teóricas provenientes de centros académicos y universidades angloeuropeas (cf. Oyuela-Caicedo et al. 1997, Pagán-Jimenez 2004); ganando también adeptos al norte del Río Grande, sin embargo, funcionó más bien como un bloque ideológico ante un avance incesante de modelos capitalistas y el florecimiento de gobiernos militares en Latinoamérica, auspiciados por el andamiaje político de la guerra fría, y perdió vigencia al final de la misma (Angelo 2005).

La crítica a la naturaleza colonial de la disciplina vendría de la mano de otra generación de la arqueología latinoamericana que hacía eco de la crítica global al carácter colonialista de la arqueología, denunciando alianzas excluyentes con proyectos nacionalistas e incorporando temáticas como la representación del pasado, marginalidad histórica de pueblos indígenas y otros (Gnecco 1999, Politis 2001; Kojan y Angelo 2005; cf. Gosden 1999). Nuestro aporte al debate, desde Latino América, sin embargo, pareciera condensarse más en torno a discusiones con respecto a problemáticas del nacionalismo, representación de pueblos indígenas y aspectos políticos relacionados a contextos históricos particulares. Hasta aquí, al parecer y salvo escasas excepciones, la academia convencional (occidental europea) ha estado poco interesada en lo que colegas de otras latitudes tengan que decir o es que arqueólogas y arqueólogos de aquello que algunos definen como el «sur global» nos acogemos más bien a la cómoda postura de consumidores de conocimiento (Mizoguchi 2015:12); en cualquier caso, la ecuación final solo profundiza aún más la dicotómica división de productores y consumidores de teoría/metodología.

Sin embargo, son precisamente trabajos como los que se incluyen en este volumen del Boletín de Arqueología de la PUCP, los que dinamizan este escenario. Con esto, me apresuro en aclarar, no intento menoscabar otras importantes contribuciones de colegas al conocimiento del pasado. 
Distintos han sido los foros que buscan promover este tipo de instancias y una diversidad de volúmenes editados como resultado de talleres y conferencias dan fé de estos esfuerzos ( $v$.g. Funari et al. 1999, Lema et al. 2008; Gnecco y Ayala 2011; Rosenfeld y Bautista 2017). Además, el creciente interés por contraponer un balance a una situación de dependencia intelectual, por ejemplo, se tradujo en importantes contribuciones de colegas que buscando incorporar perspectivas «más propias», aportaron al abordaje de temáticas relevantes al contexto histórico y político de nuestras sociedades latinoamericanas (v.g. Zarankin y Acuto 1999; Funari y Zarankin 2006; Gnecco y Langebaek 2006 y otros). Sin embargo, los aportes que busquen integrar nuevos marcos teóricos en su discusión, insistiendo en propuestas que incorporen contribuciones diversas desde la propia Latinoamérica, son aún incipientes (Haber 2009, Lema y Pazzarelli 2015).

\section{Buscando conexiones}

Las contribuciones que comprenden el presente volumen derivan de la iniciativa surgida en la organización del simposio Materialidad, práctica y agencia: Re-examinando las relaciones sociales y la gestación del poder politico, dentro del octavo congreso de Teoría Arqueológica de Sur America (TAAS) que se realizara el 2016 en La Paz, Bolivia. Es altamente gratificante ver que tal esfuerzo se traduce ahora esta publicación del Boletín de Arqueología de la PUCP. Las temáticas de los textos giran en torno a la tríada paisaje, cosas y gente/cuerpos, que son considerados desde distintos ángulos y contextos histórico-geográficos, mayormente situados en Latinoamérica. A aquellos contextos de los Andes, costeros y serranos (Muro, Corcoran-Todd, y Fernandini), complementados por miradas desde la región del Tafí y las interminables pampas del sur de Córdoba, en Argentina (Franco y Mackinson), se suma la contribución de Ian Hodder, una de las figuras más conocidas por sus aportes al debate teórico, exponiendo sus reflexiones a partir de su trabajo en Çatalhöyuk, Turquía.

Los temas en este volumen — como dice su nombre-, se entrelazan entre el paisaje, cosas y cuerpos en contextos temporales y geografías diversas; estos son analizados a partir de lentes teóricos que enfatizan la importancia de introducir nuevas conceptualizaciones de lo material y las relaciones que se establecen entre las cosas y los seres humanos. Apoyados en un andamiaje teórico que realza la importancia de superar la perspectiva antropocentrica y dicotómica que por mucho tiempo caracterizó a la disciplina, las contribuciones de este volumen son una invitación a re-abrir debates que prometen ser fructíferos. La antropología y la arqueología se interesaron desde muy temprano por las distintas relaciones de alteridad que diferentes pueblos de los Andes mantienen con su entorno material, consideradas como la esencia de lo que en su momento se llamó «cosmología andina» «reciprocidad andina» o "lo andino». Así, diferentes trabajos etnográficos documentaron estas relaciones y enmarcaron sus interpretaciones en términos substantivistas (en relación con la economía), sistémico-funcionalistas (en cuanto a conceptualizationes territoriales y uso del espacio) o de soslayado tono neo-evolucionista (en la conceptualización animística de estas relaciones) (Murra 1975; Isbell 1977; Mayer 1979).

Recientemente, aportando al debate propuesto por el denominado giro ontológico y de los nuevos materialismos (Bennett 2009; Latour 2005; Viveiros de Castro 1998, 2004; cf. Kohn 2015), trabajos como los de Marisol de La Cadena (2015) enfatizan la necesidad de ampliar la agenda conceptual de la antropología (andina) para entender dichas relaciones superando la visión antropocéntrica y dicotómica sujeto/objeto, cultura/naturaleza antes implícita. Quizás es importante aclarar que el giro ontológico, como otras corrientes de pensamiento que la precedieron, no conforma un cuerpo teórico homogéneo sino que se constituye a partir de contribuciones de disciplinas de las humanidades, como de STS y la filosofía; así, el giro ontológico ha sido comúnmente representado en posturas o etiquetas, entre las que se pueden mencionar: el perspectivismo; la relacionalidad; la Teoría del Actor-Acción; teoría del ensamblaje; la teoría del entrelazamiento (entanglement) (Kohn 2015). A riesgo de colapsar y minimizar las diferencias y énfasis que cada una de las autoras y autores de estas propuestas y el argumento de las personas que contribuyen a 
este volumen, me atrevo a decir que es precisamente el interés implícito en todas y cada una de las variantes mencionadas (insatisfacción con discursos antropocéntricos, cuestionamiento a dimensiones lineales de temporalidad o problematización del esquema colonial cultura/naturaleza), lo que constituye el principal aglutinador de este volumen.

Buscando superar las contradicciones que el discurso constructivista había promovido, en la arqueología, la disciplina de las cosas (Olsen 2007, 2010), el giro ontológico tuvo una buena, aunque no problemática, recepción ( $c f$. Thomas 2015). La influencia de autores como Viveiros Castro y Latour ha sido significativa en la arqueología global (Alberti et al. (eds.) 2013; Watts (ed.) 2014); sin embargo, se podría decir que los estudios de caso latinoamericanos en los que sus propuestas son consideradas, son aún escasos (ver, por ejemplo, Bray 2009; Haber 2009; Laguens 2013; Alberti 2014; Lema y Pazzarelli 2015; entre otros) y, en algunos casos, han sido recibidos con escepticismo (Swenson 2015; Weismantel 2015). Si bien estas nuevas corrientes de pensamiento (neo-materialismos) están basadas en algunos fundamentos planteados ya desde el materialismo crítico e histórico, como bien dice Corcoran-Tadd en este volumen, su principal aporte radica en las implicaciones éticas y políticas, además de epistemológicas, de su análisis.

\section{Convergencias (y divergencias)}

Por mucho tiempo, la arqueología andina (y principalmente la peruana), mantuvo una vinculación firme con aquellas corrientes teóricas de la arqueología procesual que, buscando proveer una explicación científica del pasado, recurrió a modelos y esquemas, prevalentemente funcional-evolutivos, en los que las sociedades prehispánicas eran consideradas. El caso del estudio de sociedades como los moche de la costa norte del actual Perú que Luis Muro aborda en este volumen, es precisamente un ejemplo en el que aspectos como ritualidad y simbología fueron generalmente contemplados como funcionales a ideologías de poder establecidas, en marcos sociales de tipo jerárquico por parte de elites religiosa-militares (Demarrais et al. 1996; Bawden 2001; Castillo y Uceda 2008). Entre algunos elementos más llamativos e importantes relevados por diferentes autores, los moche están asociados a una compleja producción artística y ritual expresada en objetos cerámicos y pinturas murales que caracterizan algunos de los sitios arqueológicos más importantes atribuidos a esta sociedad (Castillo y Uceda 2008, Larco Hoyle 1944, Kaulicke 1992).

Ante este escenario, el texto de Muro apuesta por mostrar una lectura alternativa en la que «se incorporen» los cuerpos. San José del Moro, nos dice, es el escenario ideal para explorar fronteras simbólicas y reales del mundo Moche a través del perspectivismo, introduciendo además reflexiones sobre el cuerpo, un tema poco teorizado en la arqueología de lo Moche (sin embargo, ver Hill 2000; Swenson 2012; Weismantel 2012). Desafiando algunas de las limitaciones del perspectivismo en su aplicación a la arqueología (Alberti 2014; Santos Granero 2009; Weismantel 2015), Muro insiste en explorar su potencial uso en su análisis de la relación entre humanos y cosas. Debo remarcar, una vez más, que Muro no se limita al cuerpo humano cuando se refiere a los ancestros, al menos no al cadaver decadente al que la muerte de la ontología occidental cristiana y moderna nos tiene acostumbrados, sino que invita a considerar también sus desplazamientos y representaciones en las que, según entiendo, se produciría un proceso de recorporeización híbrida de estos ancestros que el autor interpreta como simulacra. Estos cuerpos, no obstante, exceden corporalidades individuales; esquivando la noción que la figura de autoridad ritual generalmente encarnada en dignatarios moche, Muro se interesa en las relaciones del proceso que él identifica como la construcción de los ancestros. Ciertamente, los ancestros de los que Muro está hablando no están representados por la imagen que, favorecida ampliamente por las condiciones de conservación provistas por el medio, ha caracterizado la espectacularidad la arqueología peruana: el señor moche yaciente en medio del ajuar exquisito que concita el interés masivo en revistas de difusión como National Geographic u otras. Los ancestros que Muro nos presenta se muestran más como parte del proceso fractal, 
probablemente continuo y repetitivo, que resulta de acciones de destrucción y estabilización; de la partición o desmembramiento y quema de cuerpos, como de su re ensamblaje y endurecimiento, producto de la misma quema. Así, el planteamiento de cuerpos habitando y moviéndose entre mundos es provocador y nos brinda la posibilidad de acercarnos a formas de concebir esta conexión entre la vida y la muerte a partir de, y entre, los cuerpos de los seres involucrados en relaciones más flexibles y permeables (o liminales). De esta manera, el autor lleva la interpretación de espacios rituales moche más allá del simbolismo y la funcionalidad metafórica que comúnmente deja de lado lo físico y lo corpóreo. Los resultados iniciales presentes en su texto son prometedores; considero, por ejemplo, que la idea respecto al fuego, evidenciado en los pisos de la estructura arquitectónica observada, como un mediador fundamental en el proceso de otorgar el aliento de vitalidad a la materia, necesita ser profundizada en futuros trabajos.

El interés en la materialidad de los ancestros es uno de los nexos que articula el trabajo de Valeria Franco con el de Muro. Ambos casos comparten también resabios de una misma mirada epistemológica respecto del pasado; al igual que en el caso Moche, el recurrir a esquemas evolutivo-funcionalistas para explicar el desarrollo social de esta región caracterizó a la arqueología del noroeste argentino por muchas décadas (Haber 2006, 2012; Laguens 2006). «Durante varias décadas —dice la autora- las poblaciones prehispánicas se representaron como lanzadas a una carrera de obstáculos donde cada "salto" les proporcionaba mayor complejidad y desigualdad terminando en la meta, esto es, la dominación colonial. El pasado se definió en base a prácticas sociales despolitizadas analizándose casos de estudio que se presentaban como no igualitarios, jerárquicos, centralistas y crecientemente estatistas» (Franco, este volúmen; cursivas mías).

Así, analizando el caso de las sociedades del valle del Tafí, en el noroeste argentino, Franco plantea un cuestionamiento a modelos sociales evolutivos y la temporalidad lineal expresada en las cronologías arqueológicas a través de una aproximación distinta, guiada por una mirada de la materialidad implícita en las temporalidades, duraciones y agencias que evidencia en el registro arqueológico. Evidentemente, como lo afirma la autora, el esquema evolutivo de sociedades igualitarias que transitan a complejas, ampliamente criticado (ver por ejemplo varios trabajos en la compilación de Gnecco y Langebaek 2006), pero aún vigente en la arqueología (¡no solo Latinoamericana!), parece insuficiente al momento de su aplicación a las sociedades prehispánicas de la región del Tafí. Para el caso de las sociedades prehispánicas del Tafí, la capacidad agencial de distintas entidades y seres que parecen haber poblado su universo cosmológico, fueron comúnmente ignoradas en favor de modelos evolutivos de complejidad social.

Como se verá más adelante (ver también Hodder en este volumen), las tipologías neo-evolutivas a las que se hace referencia ofuscan escenarios más dinámicos donde la integración política y co-existencia de capitales y poderes están constituidos y negociados a través de (o quizás sea mejor decir en alianza con) agentes ancestrales que, según entiendo, es el argumento de Franco. Es importante notar, sin embargo, que la continuidad de estos ancestros — según la autora — es temporalmente variable e históricamente contingente. Esto en mi opinión, abre interesantes posibilidades de conceptualizar lo político en arqueología.

Por su parte, Francesca Fernandini presenta la aplicación de la propuesta de entrelazamientos, planteada por Hodder (2012), al contexto del sitio prehispánico Cerro de Oro. Su trabajo propone el seguimiento relacional de tres cosas: 1 ) un edificio monumental presente en ese asentamiento urbano; 2) el maíz, alimento y elemento vital presente ubicuamente en el registro arqueológico de las sociedades andinas, y 3) la cerámica, objetos vinculados a esferas domésticas, públicas y rituales. Para la autora, estas cosas «solo existen insertas dentro de una trama de relaciones donde no pueden ser aisladas de su presencia en distintas prácticas materiales» (pace Laguens y Pazzarelli). Así, embarcada en un proceso de rastreo de los hilos conectores que vinculan estas cosas, entretejiendo la vida cotidiana de la población que ocupó Cerro de Oro durante el denominado Intermedio Temprano (550-850 AD) (Ruales 2001; Fernandini 2015), las intervenciones de Fernandini nos presentan 
una compleja historia de construcción del entorno. El espacio donde se asienta el edificio monumental, antes ocupado por un grupo reducido, nos dice, es previamente desocupado y embebido de significancia, representado por la deposición de un ser humano no-nato en una de las esquinas. Según la autora, la planificación de la construcción misma se hace evidente en el registro material, dando lugar así al escenario del relato en el que Fernardini introduce a sus ocupantes, primero en una ocupación efímera que termina con el sellado (tapado y quema) de los espacios de ocupación, seguida luego de una ampliación del espacio y nuevas construcciones que son interpretadas como resultado del crecimiento demográfico del lugar ( $c f$. Fernandini 2015). A diferencia del contexto analizado por Franco, Fernandini enfatiza las dimensiones monumentales de las construcciones (aquella que ella decide seguir y las aledańas), en ambos casos, no obstante, el paisaje es el resultado de entretejimientos materiales e ideológicos. Esto se complementaría con el desarrollo de distintas otras actividades que habrían tenido lugar en los alrededores del edificio monumental, como la producción, procesamiento y consumo de bienes, notoriamente el maíz (ubicuo entre el consumo doméstico y de festines o ceremonias públicas), y las vasijas cerámicas que facilitaban este consumo. Estas vasijas, al igual que los revoques de fachadas, están hechas de material de la zona o cercanas al sitio, que - al igual que los campos agrícolas - corroboran el argumento de Fernandini respecto a las distintas materialidades y dependencias que se van estableciendo; en cierto punto, sin embargo, la intrusión de influencias foráneas deriva en la reconfiguración de estas relaciones. Entender los cambios que se producen a partir de estos procesos de hibridación, más allá de solo los productos híbridos, es un desafío llamativo que propone la autora.

El trabajo de Noa Corcoran-Tadd rescata varios de los puntos antes mencionados e hilvana estas problemáticas en su mirada a la arqueología de la colonia y los procesos de explotación de la plata en el Perú histórico. A pesar de recientes contribuciones de importancia, presentadas, por ejemplo, en los números 20 y 21 del Boletín de Arqueología de la PUCP, la arqueología histórica ha sido relegada a un segundo plano en la arqueología de los Andes, interesada más en el pasado remoto (cf. VanValkenburgh et al. 2016; Weaber et al. 2016); la presencia de evidencias arqueológicas prehispánicas de porte monumental y rica cultura material, usualmente presente en óptimas condiciones de conservación, y la vinculación del discurso arqueológico con posturas nacionalistas que ensalzaban el pasado remoto, desplazó el interés de la arqueología andina en períodos coloniales, republicanos o contemporáneos (Angelo 2017). Enfocado en el yermo paisaje del sur peruano que proveyó el entorno en el que se dibujaron los senderos y paisajes de la exportación de la plata coloniales, Corcoran-Tadd plantea cuestionamientos que involucran tanto la interpretación como la práctica de la arqueología a través del marco de la ecología política (Bennett 2009). La aproximación que Corcoran-Tadd tiene a las «nuevas materialidades», sin embargo, es cauta y sopesa cuidadosamente las críticas que esta tendencia u orientación teórica ha levantado. Los cuestionamientos que plantea se alinean a aquellas críticas poshumanistas, abordando un período histórico completamente distinto y, sin embargo, están en directa conversación con el resto de los textos presentes en este volumen. Su preocupación gira en torno a la representación de la realidad, una realidad compleja y compuesta por diferentes agentes y actantes entre los cuales los seres humanos solo son unos más, lo cual conlleva el desafío de decidir los niveles de escala y complejidad (por supuesto, este desafío conversa directamente con las otras contribuciones del volumen). Una representación tal debería considerar críticamente las jerarquías existentes y redefinirlas con relación a las clases y topologías de sus relaciones, garantizando la diversidad presente; dicha representación, remarca, es necesaria más en el sentido político que en el estrictamente representacional. «¿Cuáles son las cosas que importan? ¿En quién (y dónde nos) apoyamos? ¿Qué actores están representados?»; con estas preguntas como parte de su objetivo, Corcoran-Tadd revisa una de las regiones marginales en la arqueología de los Andes, histórica como geopolíticamente hablando, la sierra de Tacna, al sur del Perú, territorio de vital importancia para comercialización de la plata durante tiempos coloniales. Mediante el recurso fenomenológico como parte de su metodología, Corcoran-Tadd 
experimenta el paisaje recorrido por llamas, mulas y sirvientes encargados de transportar el metal en ese paisaje yermo y alturas donde falta el oxígeno, sujetos no solo a sus inclemencias sino a los sistemas de explotación colonial. En partes, este trayecto vital en la economía global, se transforma para acoger humanos y no humanos, con tambos y corrales, abrevaderos y terrazados, con alfalfa y patios, algunos con capas de estiércol, y tapiados que abrigan y confortan, no solo históricamente sino aún en el presente. En este contexto, la categoría universal del cuerpo se quiebra; humanos y no humanos conforman jerarquías según su capacidad de sobrellevar carga, marcando en algunos de ellos sus efectos de explotación colonial (DeFrance 2010). Estos entrelazamientos (CorcoranTadd no usa el término y quizás no esté de acuerdo con mi atrevimiento), producen un paisaje que quiebra la armonía histórica, ofreciendo narrativas más crudas que develan la crueldad de un sistema (y una forma de contar la historia) de explotación que somete humanos y no humanos por igual.

La contribución de María Mackinson «rompe», de alguna forma, cierta armonía arqueológica de los textos anteriores, introduciendo una aproximación a un paisaje peculiar: el cementerio de Huinca Renancó, un poblado que revela, desde su nombre («el pozo del agua del blanco», en lengua ranquel), relaciones de colonialidad presentes en su historia (...es «hijo de la Conquista del Desierto", nos dice la autora en un pie de página). El trabajo de Mackinson rompe también con la estructura usual de los textos a los que estamos acostumbrados en la retórica arqueológica disciplinar: antecedentes, argumento, conclusiones... y transita subtextos que develan presencias (la de ella, la de su abuela, la de María A.) como también sus ausencias, dejando rastros en su paso. La autora busca inquietar, o quizás solo refleja su ansiedad, mostrando ciertos límites en nuestro entender (moderno y, mayoritariamente, occidental) del mundo. Sus recorridos y permanencia en el cementerio de Huinca Renancó conducen a la autora a plantear un cuestionamiento a la prevalencia de las ideas (mente y lo visual) sobre otras formas de acceso al mundo y - a través de su cuerpo-, interroga la otredad última marcada por la muerte. Reflexionando en su trabajo etnográfico, Mackinson nos presenta una narrativa peculiar, que es presentado en un estilo coloquial que, irónicamente, se vuelve hermético en partes, en su elección de palabras, conceptos y herramientas de la escritura (tachados y vacios) a los que recurre.

De esta forma, el texto de Mackinson plantea desafíos a la lectura lineal, y sus estrategias pueden ser, en ciertos casos, contraproducentes al momento de buscar un mensaje final; o quizás, simplemente, no hay un mensaje final que se intente. Su trabajo, definitivamente más autoetnográfico que arqueológico, busca inquietar, o quizás solo refleja su ansiedad, mostrando ciertos límites en nuestro entender (moderno y, mayoritariamente, occidental) del mundo. Inspirado en distintas contribuciones de antropólogos (Ingold, Auge y otros), Mackinson adopta un lenguaje de relato activo y presente (un presente cargado de pasados) para retratar su transitar (como cuerpo/entidad sintiente) entre distintos espacios. De manera interesante, y en cierta forma contradictoria, Mackinson cuestiona lo visual como la puerta de las ideas, o al menos como sentido ordenador, pero es lo visual por donde su aproximación a lo material se produce de manera más intensa, aunque no exclusivamente; el mundo que fuga en multiplicidades, nos dice, solo parece tener sentido a partir de su experiencia. En ese sentido, Mackinson da cuenta de esos objetos/mundo que marcan y que son reportados/ observados en casos previos, en un contexto moderno.

Finalmente, el texto de Ian Hodder cierra este volumen. Asociado al movimiento post postprocesual que provocó un remezón en la teoría arqueológica, Hodder no necesita presentación. Julian Thomas describió su trabajo más reciente como: «un sofisticado argumento respecto de cómo las gentes y las cosas se han entrelazado progresivamente entre sí» (Thomas 2015: 1289) produciendo dependencias y codependencias con las cosas, en su búsqueda incesante de estabilidad y mantenimiento. En este volumen, Hodder presenta una síntesis de su libro Entanglement (2012) — y un preámbulo a su último libro Where are we heading? (2018)_, a través de la cual despliega una mirada crítica a visiones lineales y unidireccionales de desarrollo de las que, iróni- 
camente, busca desentralazar la noción de evolución, sacudiendo el lastre colonial que el término desarrollo le impuso. Así, reflexionando en torno a teorías del progreso, evolución biológica y complejidad, comúnmente presentes en narrativas arqueológicas, Hodder resalta las ventajas de su propuesta de entrelazamientos. Desplegando una estrategia similar a la observada en la contribución de Fernandini, Hodder ha ofrecido ejemplos arqueológicos que evidencian su propuesta en otras publicaciones (Hodder 2016; Hodder y Mol 2016) por lo que el publico lector interesado deberá referirse a esos textos para revisarlos. Hodder inicia su texto con una crítica a esa historia lenta y gradual, orientada a la legitimación de intereses dominantes promulgados a partir de una idea lineal de progreso en la evolución humana, que resuena con otras contribuciones en este volumen (Muro, Franco y Fernandini; $c f$. Hodder 2018). Revelando su carácter antropocéntrico, esta narrativa unidireccional, dice Hodder, «le adjudica a la especie humana una naturaleza inherente, esto es, la de siempre querer progreso» (este volumen). Por esto, continúa, es importante introducir direcciones que no estén centradas en lo humano y que presten mayor atención a las cosas (entendiendo como «cosas» el resultado de implicaciones y entrampamientos que se producen entre humanos, el mundo material —en el que incluye ampliamente lo orgánico y no orgánico — y el de las ideas) ( $c f$. Hodder 2012). Su apuesta es enfatizar la importancia a estas cosas en el proceso de redireccionar nuestro conocimiento de estos entrampamientos y entrelazamientos en un proceso de larga duración que aún continua.

\section{Pensando «en voz alta...»: la urgencia de relecturas conceptuales}

Ofrecer una síntesis abarcadora de todas las propuestas de este volumen, si quizás es feasible, es prácticamente poco o nada deseable. Contrariamente al interés de aquellos estudios comparativos culturales, pensaría que la contribución principal de este compendio radica en el desafío de incorporar nuevos elementos de análisis que, desplazando los énfasis habituales de escrutinio del pasado (y el presente), promueven conversaciones entre mundos que emergen nuevos. No obstante, a modo de abrir nuevas preguntas, es quizás importante remarcar ciertas convergencias; particularmente en lo que toca al objetivo de pensar la arqueología andina/latinoamericana. En un paréntesis importante, es necesario mencionar que, si bien en mi texto resalté autores latinoamericanos, en una arqueología que me gusta pensar como latinoamericana, no es posible dejar de lado las colaboraciones y debates que se producen gracias al intercambio de ideas con colegas de otras latitudes (como las contribuciones de Corcoran-Tadd y Hodder en este volumen).

Recientemente, respondiendo al avance rampante del neoliberalismo que afecta directamente la práctica arqueológica, Gonzalez-Ruibal, Alonso González y Criado-Boado, advierten la urgente necesidad de un rearme teórico de la arqueología que pueda hacer frente a un capitalismo que ya no necesita de un discurso científico para legitimarse, trasladando así el campo de batalla a enfrentar aparatos políticos, económicos e ideológicos (González-Ruibal et al. 2018:526 cf. Gonzalez-Ruibal 2013, Haber 2012, Hernando 2012). En ese sentido, quiero remarcar el trabajo de Corcoran-Tadd y su preocupación en visibilizar presencias olvidadas, a partir de preguntas importantes y persistentes acerca las relaciones entre la práctica, la representación y la ética. De igual forma, a pesar de las diferencias geográficas y temporales en las que los distintos ejemplos se enfocan, las conversaciones (y entrelazamientos de devenires históricos) entre los distintos trabajos aquí presentes, por ejemplo entre el Cerro de Oro y los montículos del valle del Tafí, o los ancestros de estos últimos y aquellos de San José del Moro, parecen mostrarnos la necesidad (y posibilidad) de escapar de esquemas rígidos (y tiránicos) que, además, condicionaban a estos mismos devenires en términos de un desarrollo lineal (cf. Gnecco y Langebeck 2006).

Dentro de la propuesta decolonial, Haber $(2006,2012)$ argumenta que uno de los campos de batalla más importantes es el del tiempo y las temporalidades que la arqueología construye en su andamiaje teórico (por ejemplo, la imagen de un pasado compartamentalizado por cronologías 
que responden a marcos unidireccionales). El rol que la arqueología jugó en el establecimiento de la brecha metafísica que irrumpe y corta aquellos relacionamientos, o entrelazamientos (sensu Fernandini, este volumen) que estructuraban las sociedades prehispánicas, es tan importante como el proceso de extirpación de idolatrías durante la colonia ( $c f$. Haber 2009). Los cuestionamientos políticos — relacionado a políticas identitarias, reivindicaciones étnicas y de valoración cultural— constituyen avances en esta línea. No obstante, muchos objetores de este avance, aceptaron la intrusion de aspectos politicos identitarios, pero mantuvieron el pasado despoliticizado (jel pasado tenía que ser objetivo y empíricamente comprobable!). En ese sentido, otro de los entrelazamientos que celebro es precisamente la combinación de una crítica teórica a esquemas usualmente dominantes mediante la incorporación de elementos que, claramente, facilitan un acercamiento a otras dimensiones más amplias e interesantes de la vida social de las sociedades prehispánicas.

Los trabajos de Fernandini, Franco y Muro, en ese sentido, enfatizan la necesidad de considerar esquemas que escapen a aquellos modelos organicistas en los que términos como complejidad, jerarquía y orden social están enmarcados en análisis unidimensionales, tanto cronológica como espacialmente. Franco y Muro ejemplifican esta problemática y nos recuerdan que un cuestionamiento teórico-político, para ser efectivo, también necesita plantearse en el pasado, o en las representaciones del mismo. De igual forma, las reflexiones que Muro ofrece respecto de las relacionalidades que se producen a través de ancestros que crean y recrean, y las de Mackinson en torno a la conceptualización de los cuerpos que son capaces de componer (en ellos) otros cuerpos en devenires que se producen en su construcción, invitan a repensar ontologías rígidas como aquella definida por el binario masculino y femenino o el de biológico y cultural (y otros: sexo/género, mente/cuerpo, etc.); recientes oleadas de críticas feministas planteadas mayormente por generaciones latinoamericanas jóvenes, demuestran que muchas de estas categorías no solo siguen constrińendo nuestras interpretaciones arqueológicas ( $c f$. Joyce 2005; Marshall y Alberdi 2014), sino la forma en la que los cuerpos son pensados en el presente.

La reciente implementación de marcos teóricos neomaterialistas (u otros) en el contexto andino (o cualquier otro) no está extenta de desafíos. En una reciente publicación, Weismantel ha sido crítica de una adopción rápida y feliz (¡y poco reflexiva!) de la corriente perspectivista en la arqueología andina (2015); el desafío que Weismantel plantea para afrontar tal problema es reconocer y orientar nuestras investigaciones para que estas tengan implicancias en nuestras sociedades (Weismantel 2015:17). Por otra parte, valorando el aporte del giro ontológico en la arqueología, Swenson plantea que la revisión de ontologías alternativas, como la Amerindia (Viveiros de Castro 2004 y otros), «debería servir no solo para cambiar aquellas preocupaciones tradicionales de la antropología para con la cultura, la práctica y la ideología, sino para reforzarlas» (Swenson 2015: 678). Para Swenson, la aplicación de estos aportes teóricos debería ir más allá de «confirmar si la ontología de una sociedad Andina valida la teoría del meshwork de Ingold o bien las de la ANT de Latour». Uno solo puede asentir a tal afirmación; quizás solo añadiría también la necesidad de establecer tratamientos comparativos, que no estén orientados a comparaciones culturales, sino a facilitar la emergencia y encuentros de "mundos dentro del mundo» en un plano que no sea el establecido por la epistemología colonial.

\section{«El cadaver exquisito beberá la copa del vino nuevo»}

Considero importante remarcar la apertura del Boletín de Arqueología de la PUCP, una de las publicaciones más prestigiosas de la arqueología peruana, a contribuciones que arriesguen ejercicios teóricos. La intensa e incesante dinámica global ha modificado los tradicionales centros de producción de conocimiento; como reconoce Olsen, «a pesar del hecho de que editoriales angloamericanas todavía mantienen un gran porcentaje en la publicación internacional relacionado a la arqueología (tanto en libros como en revistas especializadas) y, a pesar de que [el idioma] inglés es más fuerte que nunca, lo que emerge y continuará emergiendo es un paisaje arqueológico menos centralizado 
en las metrópolis tradicionales de conocimiento» (Olsen 2012: 19). Foros como el Congreso de Teoría Arqueológica de Sur América han sido posiblemente los que más visibilizaron estos esfuerzos, pero ciertamente otros espacios, como aquellos que se promueven periódicamente en los distintos países, han sido altamente efectivos en promover dicha sinergia en un mundo global de colaboraciones Norte-Sur, Sur-Sur (los ejemplos aquí podrían desbordar el texto).

En el desarrollo de un pensamiento teórico que responda a nuestros contextos, me parece importante valorar las contribuciones, principalmente aquellas que se producen en espacios y por autores latinoamericanos, de modo tal que podamos remontar ese desequilibrio en términos de la producción y consumo de teoría arqueológica. En otras palabras: cuando toque consumir teoría, consuma (también) lo nuestro. Quizás no sea necesario mencionarlo, pero autores latinoamericanos han contribuido significativamente a la discusión de temáticas como fronteras, desarrollo, dependencia, etc. Una revisión del tema excede los límites de este texto, pero es importante mencionar que algunas de las contribuciones más importantes a estos temas fueron planteadas desde contextos y espacios de análisis latinoamericanos (v.g. Zea 1986; Dussel 1994; Lins Ribeiro 1994; Escobar 2011), y proveerian un interesante contrapunto a las discusiones en torno a progreso y desarrollo que plantea Hodder en su texto (cf. Gnecco y Dias 2015).

Como parte de ese esfuerzo quizás sea necesario el producir nuevos lenguajes y términos (Haber 2012) y estrategias retóricas que recurra a técnicas exploradas en la literatura u otras para lograr tales objetivos. La importancia de incorporar estas nuevas reconfiguraciones al lenguaje interpretativo y emplear la retórica como un elemento importante en la crítica y la acción política ha sido remarcada recientemente por González-Ruibal (2013b). El texto de Mackinson, quién utiliza varios mecanismos en su presentación —al estilo de la técnica de recorte y doblez que William Burroughs popularizara en algún momento- , rompe la linealidad del relato y plantea ciertas incertidumbres al momento de considerar aspectos que, según la autora, nos marcan en la relación objetos-mundo, es quizás un buen ejemplo en este volumen.

Finalmente, insisto en la necesidad de pensar la arqueología como intrínsecamente ligada al presente. En ese sentido, comparto plenamente la apreciación de Hodder que «la arqueología dejó de ser el estudio del pasado a través de los restos materiales — frase con la que inicio este texto-, para pasar a ser el proceso de estudiar las relaciones entre la gente y sus pasados materiales [...] siempre intrusivo, siempre una intervención» (Rathje et al. 2013: 123). Citando a Coole y Frost, y Jane Bennett, Corcoran-Tadd dice: «los neo-materialistas han recurrido a las vitalidades [...] y el reencantamiento [...] como bases para intervenciones que amplien el compromiso de actores politicos y alienten nuevas sensibilidades afectivas hacia este compromiso» (este volumen, mis cursivas). La arqueología tiene un aporte transformativo que se produce en la práctica, en el proceso de producción de saber y la compilación de evidencias (mapas, fotos, colecciones, etc.); «rasgos arqueológicos, artefactos y sitios forman conjuntos que devienen nuevas formas a través de estos modos activos, e incluso performativos, de producción y transformación (Shanks 2004), más que existir como objetos pasivos e inertes», dice Corcoran-Tadd (este volumen).

Ciertamente, la revisión de trabajos como los de Nadia Abu El-Haj (2001), y aquellos referidos al rol de los monumentos en la validación del discurso nacionalista y patriarcal, le darían la razón. Desafortunadamente, como argumentan González-Ruibal y colegas (2018a) los desafíos se multiplican desde diferentes frentes. Mientras termino de escribir este texto —a través de la ventana global - soy testigo lejano de un revuelo en la internet suscitada por la reciente reactivación del debate sobre «momias extraterrestres». Los cuerpos de estos supuestos alienígenas, originalmente presentados en el congreso mundial de momias el 2017, fueron - luego de evaluaciones cuidadosas - rápidamente probadas como fraude. Sin embargo, luego de un año del suceso, el lobby realizado promovía un nuevo debate en una prestigiosa universidad peruana y la posibilidad de que el congreso de ese país apruebe un proyecto de ley para investigarlas (https://elcomercio.pe/ tecnologia/ciencias/proyecto-ley-quiere-declarar-interes-historico-supuestas-momias-humanoides- 
noticia-579622). Lamentablemente, este no es el único caso de recientes intentos de apropiarse del pasado, en desmedro de la gente y las cosas que lo constituyen, mientras ganan popularidad entre el público y denostan a los herederos de esos pasados ( $c f$. Alonso Gonzalez 2016). Habría que pensar en cómo actuar performativamente, junto a estos materiales, en una práctica que afronte estos y otros desafíos y que impulse el cambio.

\section{Agradecimientos}

Quiero expresar mi gratitud a Francesca Fernandini, Luis Muro y al comité editor del Boletín de Arqueología PUCP por su gentil invitación y por su paciencia.

\section{REFERENCIAS}

Abu El-Haj, N.

2001 Facts on the ground: Archaeological practice and territorial self-fashioning in Israeli society, Chicago University Press, Chicago.

Alberti, B.

2014 Designing body-pots in the Formative La Candelaria culture, northwest Argentina, en: E. Hallam y T. Ingold (eds.), Making and growing: Anthropological studies of organisms and artefacts, 107-125, Ashgate, Farnham.

Alberti, B., A. M. Jones y J. Pollard (eds.)

2013 Archaeology after interpretation: returning materials to archaeological theory, Left Coast Press, Walnut Creek.

Alonso González, P.

2016 Between certainty and trust: Boundary-work and the construction of archaeological epistemic authority, Cultural Sociology 10(4), 483-501. https://doi.org/10.1177/1749975516640569

Angelo, D.

2005 La arqueología boliviana: reflexiones sobre la disciplina a inicios del siglo XXI, Arqueología Suramericana 1(2), 185-211.

Angelo, D.

2017 Histories of a burnt house: An archaeology of negative spaces and dispossession, American Anthropologist 119(2), 253-268. https://doi.org/10.1111/aman.12868

Bate, L. F.

1977 Arqueología y materialismo histórico, Ediciones de Cultura Popular, México DF.

Bate, L. F. y A. Terrazas, A.

2002 Sobre el modo de reproducción en sociedad pretribales, Revista Atlantica-Mediterranea de Prehistoria y Arqueología Social 5(1), 11-41. https://doi.org/10.25267/Rev_atl-mediterr_prehist_arqueol_soc.2002. v5.01

Bawden, G.

2001 The symbols of late Moche social transformation, Studies in the History of Art 63, 284-305.

Bennett, J.

2009 Vibrant matter: A political ecology of things, Duke University Press, Durham. https://doi. org/10.1215/9780822391623

Binford, L. R.

1983 In pursuit of the Past, Thames and Hudson, Londres.

1987 Data, relativism and archaeological science, Man, 22(3), 391-404. https://doi.org/10.2307/2802497 
Bray, T. L.

2009 An archaeological perspective on the Andean concept of camaquen: Thinking through late pre-Columbian ofrendas and huacas, Cambridge Archaeological Journal 19(03), 357-366. https://doi.org/10.1017/ S0959774309000547

\section{Castillo Butters, L. y S. Uceda}

2008 The mochicas, en: H. Silverman y W. Isbell (eds.), The handbook of South American archaeology, 707-729, Springer, Nueva York.

DeFrance, $\mathrm{S}$.

2010 Paleopathology and health of native and introduced animals on southern Peruvian and Bolivian Spanish Colonial sites, International Journal of Osteoarchaeology 20, 508-524. https://doi.org/10.1002/oa.1074

De la Cadena, $M$.

2015 Earth beings: Provincializing nature and the human through Andean worlds, Duke University Press, Durham. https://doi.org/10.1215/9780822375265

DeMarrais, E., L. J. Castillo y T. Earle

1996 Ideology, materialization, and power strategies, Current Anthropology 37(1), 15-31. https://doi. org/10.1086/204472

Dussel, E.

1994 1492: El encubrimiento del otro: hacia el origen del «mito de la modernidad», Plural, La Paz.

Escobar, A.

2011 Encountering development: The making and unmaking of the Third World, Princeton University Press, Durham.

Fernandini, F. G.

2015 Beyond the empire: Living in Cerro de Oro, tesis de doctorado, Departamento de Antropología, Stanford University.

Funari, P., E. Goes Neves e I. Podgorny

1999 Introduçáo - A primeira reunião internacional de teoria arqueológica na América do Sul: questôes e debates, Revista do Museu de Arqueologia e Etnologia, (3), 1-12.

Funari, P. y A. Zarankin

2006 Arqueología de la represión y la resistencia en América Latina (1960-1980), Editorial Brujas, Córdoba.

Gándara, M.

1987 Hacia una teoría de la observación en arqueología, Boletín de Antropología Americana 15, 5-13.

Gándara, $M$.

1992 El análisis teórico: aplicaciones al estudio del origen de la complejidad social, Boletín de Antropología Americana 25, 93-104.

Gnecco, C.

1999 Multivocalidad histórica. Hacia una cartografía postcolonial de la arqueología, Departamento de Antropología, Universidad de Los Andes, Bogotá.

Gnecco, C. y P. Ayala

2011 Archaeology and indigenous peoples in Latin America, Left Coast Press, Walnut Creek.

Gnecco, C. y C. Langebaek

2006 Contra la tiranía tipológica en arqueología. Una visión desde Sur América, Ediciones UniAndes, Bogotá.

Gnecco, C. y A. S. Dias

2015 On contract archaeology, International Journal of Historical Archaeology 19(4), 687-698. https://doi. org/10.1007/s10761-015-0305-6

González-Ruibal, A.

2013 Reclaiming archaeology: Beyond the tropes of modernity, Routledge, Londres. https://doi. org/10.4324/9780203068632 


\section{González-Ruibal, A., P. Alonso González y F. Criado-Boado}

2018a Against reactionary populism: towards a new public archaeology, Antiquity 92(362), 507-515. https:// doi.org/10.15184/aqy.2017.227

2018b Authority vs power: capitalism, archaeology and the populist challenge, Antiquity 92(362), 525-527. https://doi.org/10.15184/aqy.2018.26

Gosden, C.

1999 Anthropology and archaeology. A changing relationship, Routledge, Londres.

Haber, A. F.

2006 Agricola est quem domus demonstrat, en: C. Gnecco y C. Langebaek (eds.), Contra la tiranía tipológica en arqueología. Una visión desde Sur América, 77-97, Universidad de Los Andes, Facultad de Ciencias Sociales, Bogotá.

2009 Animism, relatedness, life: Post-western perspectives, Cambridge Archaeological Journal 19(3), 418-430. https://doi.org/10.1017/S0959774309000602

2012 Un-disciplining archaeology, Archaeologies 8(1), 55-66. https://doi.org/10.1007/s11759-011-9178-4

Hernando, A.

2012 La fantasía de la individualidad: sobre la construcción sociohistórica del sujeto moderno, Katz Editores, Buenos Aires.

Hill, E.

2000 The embodied sacrifice, Cambridge Archaeological Journal 10(2), 317-326. https://doi.org/10.1017/ S0959774300000123

Hodder, I. (ed.)

1982 Symbolic and structural archaeology, Cambridge University Press, Cambridge. https://doi.org/10.1017/ CBO9780511558252

Hodder, I.

2012 Entangled: An archaeology of the relationships between humans and things, Wiley-Blackwell, Malden. https://doi.org/10.1002/9781118241912

2016 Degrees of dependence. The example of the introduction of pottery in the Middle East and at Catalhöyük, en: L. Dey y F. Fernandini (eds.), Archaeology of entanglement, 235-250, Routledge, Londres.

2018 Where are we heading? The evolution of humans and things, Yale University Press, New Haven.

Hodder, I. y A. Mol

2016 Network Analysis and Entanglement, Journal of Archaeological Method and Theory 23(4), 1066-1094. https://doi.org/10.1007/s10816-015-9259-6

Isbell, B. J.

1977 To defend ourselves. Ecology and ritual in an Andean village, University of Texas Press, Austin.

Joyce, R.

2005 Archaeology of the Body, Annual Review of Anthropology 34, 139-158. https://doi.org/10.1146/annurev. anthro.33.070203.143729

Kaulicke, P.

1992 Moche, Vicús y el Mochica, Bull. Inst. Fr. Études Andines 21(3), 853-903.

Kintigh, K. W., J. Altschul, M. Beaudry, R. Drennan, A. Kinzig, T. Kohler, W. Limp, H. Maschner, W. Michener, T. Pauketat, P. Peregrine, J. Sabloff, T. Wilkinson, H. Wright y M. Zeder

2014 Grand challenges for archaeology, American Antiquity 79(1), 5-24. https://doi.org/10.7183/00027316.79.1.5

Kohl, P. L.

1993 Limits to a post-processual archaeology (or, the dangers of a new scholasticism), en: N. Yoffee y A. Sherratt (eds.), Archaeological theory: who sets the agenda, 13-19, Cambridge University Press, Cambridge.

Kohn, E.

2015 Anthropology of ontologies, Annual Review of Anthropology 44, 311-327. https://doi.org/10.1146/annurev-anthro-102214-014127 
Kojan, D. y D. Angelo

2005 Dominant narratives, social violence and the practice of Bolivian archaeology, Journal of Social Archaeology 5(3), 383-408. https://doi.org/10.1177/1469605305057585

Kristiansen, K.

2014 Towards a new paradigm. The third science revolution and its possible consequences in archaeology, Current Swedish Archaeology 22(4), 11-71.

Laguens, A.

2006 Espacio social y recursos en la arqueología de la desigualdad social, en: C. Gnecco y C. Langebaek (eds.), Contra la tiranía tipológica en arqueología. Una visión desde Suramérica, 99-120, Uniandes, Bogota.

2013 Unstable contexts: relational ontologies and domestic settings in Andean northwest Argentina, en: B. Alberti, A. M. Jones, y J. Pollard (eds.), Archaeology after interpretation: returning materials to archaeological theory, 97-114, Left Coast Press, Walnut Creek.

Larco Hoyle, R.

1944 Cultura Salinar: sintesis monográfica, Chiclín.

Latour, B.

2005 Reassembling the social. An introduction to actor-network-theory, Oxford University Press, Oxford.

Lema, V. S. y F. Pazzarelli

2015 Memoria fértil. Crianza de la historia en Huachichocana. Nuevo Mundo Mundos Nuevos. Nouveaux Mondes Mondes Nouveaux-Novo Mundo Mundos Novos-New World New Worlds, http://journals.openedition.org/nuevomundo/67976, accedido en noviembre de 2018

Lema, V., S. Archila y M. Giovanetti (eds.)

2008 Arqueobotánica y teoría arqueológica. Discusiones desde Suramérica, Uniandes, Bogotá.

Lins Ribeiro, G.

1994 Transnational capitalism and hydropolitics in Argentina: The Yacyretá high dam, University Press of Florida, Gainesville.

Lumbreras, L. G.

1989 Chavin de Huántar en el nacimiento de la civilización andina, Ediciones INDEA, Lima.

2007 El Imperio Wari, Instituto Francés de Estudios Andinos, Lima.

Marshall, Y. y B. Alberti

2014 A matter of difference: Karen Barad, ontology and archaeological bodies, Cambridge Archaeological Journal 24(1), 19-36. https://doi.org/10.1017/S0959774314000067

Mayer, E.

1979 Land-use in the Andes: ecology and agriculture in the Mantaro Valley of Peru with special reference to potatoes, International Potato Center, Lima.

Mizoguchi, K.

2015 A future of archaeology, Antiquity 89(343), 12-22. https://doi.org/10.15184/aqy.2014.39

Morandé, P.

1984 Cultura y modernización en América Latina, Cuadernos del Instituto de Sociología de la Pontificia Universidad Católica de Chile, Santiago de Chile.

Murra, J.

1975 El control vertical de un máximo de pisos ecológicos en la economía de las sociedades andinas, en: J. Murra (ed.), Formaciones politicas y económicas del mundo andino, Instituto de Estudios Peruanos, Lima.

Olsen, B.

2007 Keeping things at arm's length. A genealogy of asymmetry, World Archaeology 39(4), 579-588. https:// doi.org/10.1080/00438240701679643

2010 In defense of things. Archaeology and the ontology of objects, Altamira Press, Lanham.

2012 After interpretation: Remembering archaeology, Current Swedish Archaeology 20, 11-34. 


\section{Pagán-Jiménez, J. R.}

2004 Is all archaeology at present a postcolonial one? Constructive answers from an eccentric point of view, Journal of Social Archaeology 4(2), 200-213. https://doi.org/10.1177/1469605304041075

Politis, G.

2001 On archaeological praxis, gender bias and indigenous peoples in South America, Journal of Social Archaeology 1(1), 90-107. https://doi.org/10.1177/146960530100100107

2003 The theoretical landscape and the methodological development of archaeology in Latin America, American Antiquity 68(2), 245-272. https://doi.org/10.2307/3557079

Politis, G. y J. Pérez Gollán

2004 Latin American archaeology: From colonialism to globalization, en: L. Meskell y R. Preucel (eds.), $A$ companion to social archaeology, 353-373, Blackwell, Oxford. https://doi.org/10.1002/9780470693605. ch15

Ruales, M.

2001 Investigaciones en Cerro del Oro, valle de Cañete, Boletín de Arqueología PUCP 4, 359-399.

\section{Rathje, W., M. Shanks y C. Witmore}

2013 Ian Hodder, en: W. Rathje, M. Shanks y C. Witmore (eds.), Archaeology in the making. Conversations through a discipline, 122-138, Routledge, Londres.

Sanoja, M.

1984 La inferencia en la arqueología social, Boletín de Antropología Americana 10, 35-44.

Santos-Granero, F. (ed.)

2009 The occult life of things: Native Amazonian theories of materiality and personhood, University of Arizona Press, Tucson.

Shanks, M. y C. Y. Tilley

1987 Social theory and archaeology, Polity Press, Cambridge.

Swenson, E.

2015 The materialities of place making in the ancient Andes: A critical appraisal of the ontological turn in archaeological interpretation, Journal of Archaeological Method and Theory 22(3), 677-712. https://doi. org/10.1007/s10816-014-9202-2

\section{Swenson, E. y J. Warner}

2012 Crucibles of power: Forging copper and forging subjects at the Moche Ceremonial center of Huaca Colorada, Peru, Journal of Anthropological Archaeology 31(3), 314-333. https://doi.org/10.1016/j. jaa.2012.01.010

Thomas, J.

2015 The future of archaeological theory, Antiquity 89(348), 1287-1296. https://doi.org/10.15184/ aqy. 2015.183

\section{Trigger, $\mathbf{B}$.}

1989 A History of Archaeological Thought, Cambridge University Press, Cambridge.

VanValkenburgh, P., Z. Chase, A. Arias y B. J. M. Weaver

2016 Arqueología histórica en el Perú: posibilidades y perspectivas, Boletín de Arqueología PUCP 20, 5-24. https://doi.org/10.18800/boletindearqueologiapucp.201601.001

\section{Viveiros de Castro, E.}

1998 Cosmological deixis and Amerindian perspectivism, The Journal of the Royal Anthropological Institute 4(3), 469-488. https://doi.org/10.2307/3034157

2004 Perspectival anthropology and the method of controlled equivocation, Tipiti: Journal of the Society for the Anthropology of Lowland South America 2(1), 1.

Watts, C. (ed.)

2014 Relational archaeologies: Humans, animals, things, Routledge, Londres. https://doi. org/10.4324/9780203553138 
Weaver, B. J. M., A. Traslaviña, P. VanValkenburgh y Z. J. Chase

2016 Arqueología histórica en el Perú: la sociedad andina en la transición económica, política y social, Boletín de Arqueología PUCP 21, 5-12. https://doi.org/10.18800/boletindearqueologiapucp.201602.001

Weismantel, $\mathrm{M}$.

2012 Obstinate things, en: B. L. Voss y E. Casella (eds.), The archaeology of colonialism, 303-320, Cambridge University Press, Cambridge.

2015 Seeing like an archaeologist: Viveiros de Castro at Chavín de Huantar, Journal of Social Archaeology 15(2), 139-159. https://doi.org/10.18800/boletindearqueologiapucp.201602.001

Yoffee, N. y A. Sherratt

1993 Archaeological theory: who sets the agenda? Cambridge University Press, Cambridge. https://doi. org/10.1017/CBO9780511720277

\section{Zarankin, A. y F. A. Acuto}

1999 Sed non satiata: Teoría social en la arqueología latinoamericana contemporánea, Ediciones del Tridente, Buenos Aires.

Zea, L.

1986 América Latina en sus ideas, Siglo XXI Editores, Mexico DF.

Recepción: noviembre 2018

Aceptación: diciembre 2018 